\title{
VALIDATED RP-HPLC METHOD FOR QUANTITATION OF GLIQUIDONE IN PHARMACEUTICAL FORMULATION AND HUMAN SERUM
}

\author{
M. SAEED ARAYNE ${ }^{1}$, NAJMA SULTANA², AGHA ZEESHAN MIRZA' AND FARHAN AHMED SIDDIQUI ${ }^{1}$ \\ ${ }^{1}$ Department of Chemistry, University of Karachi, Karachi-75270, Pakistan \\ ${ }^{2}$ Research Institute of Pharmaceutical Sciences, Faculty of Pharmacy, University of Karachi-75270, Pakistan
}

(Received: September 30, 2008 - Accepted: September 11, 2009)

\begin{abstract}
1. A simple isocratic reversed phase high performance liquid chromatographic (HPLC) method using C18 column with ultraviolet detection at $225 \mathrm{~nm}$ has been described for quantitative determination of gliquidone in formulation and human serum. The system is operated at room temperature using a mobile phase consisting of methanol: water (90:10) adjusted to $\mathrm{pH} 3.50$ with phosphoric acid with a flow rate of $1 \mathrm{mLmin}^{-1}$. Methylparaben is successfully used as an internal standard. The assay is reproducible, linear (concentration range of $0.60-25 \mu \mathrm{gmL}^{-1}$ ) with correlation coefficient of 0.9999 and accurate (99.23 to $102.78 \%$ ). The intra and inter run precision results are 1.14 and $1.32 \%$. The LOD and LOQ values are 0.03 and $0.6 \mu \mathrm{gmL}^{-1}$, respectively. There are no interfering peaks due to the excipients present in the pharmaceutical tablet. Thus, the proposed method is simple and suitable for the analysis of active ingredient in tablet dosage form and human serum.
\end{abstract}

Key words: Gliquidone, methylparaben, UV detection, RP-HPLC, dosage formulation, method validation.

\section{INTRODUCTION}

Sulfonylurea has been the mainstay of antidiabetic therapy for many years. Gliquidone belongs to the class of sulfonylurea derivative and is mainly indicated for the treatment of non-insulin dependent diabetes mellitus (NIDDM) (figure 1). It improves glycemic control and lowers blood sugar level by stimulating the production and release of insulin from pancreas and also promotes the movement of sugar from the blood into the required cells [1-3]. Use of this drug in patients with NIDDM having liver diseases should be restricted but no clinical or laboratory deterioration on the part of the liver have been detected over the course of follow-up, therefore the drug may be used in the treatment of NIDDM patients with liver abnormalities [4]. Literature survey revealed that quantification of gliquidone has been achieved by UV spectrophotometry [5], atmospheric pressure chemical ionization liquid chromatographic-mass spectrometric (APCI-LC-MS) LC-MS [6] and HPLC [7-9]. Most of these HPLC methods for gliquidone, involve either complicated mobile phases or buffers that may be corrosive to the column or flow system of HPLC. Guo et al. [7] reported the assay of gliquidone in plasma using HPLC column switching technique. A column switching method has the problem of the availability of the required instrument. One study [8] reported the duration of action and the pharmacokinetics of gliquidone in serum by high performance liquid chromatography with fluorescence detection. Another method in which Sridevi et al. [9] was determined gliquidone in rat plasma and conducted pharmacokinetic studies using photodiode array detector. Since all these methods requires expensive instrument and reagent. There was a need for a rapid and economical analytical method for routine analysis of gliquidone in bulk and pharmaceutical preparation. The purpose of this study was, to develop a simple and reliable method with a better detection range for analysis of gliquidone, using commercially available internal standard. The optimum UV wavelength of the present study $(225 \mathrm{~nm})$ was selected on the basis of higher sensitivity [5]. The established method was validated with respect to specificity, linearity, precision and accuracy.<smiles>COc1ccc2c(c1)C(=O)N(CCc1ccc(S(=O)(=O)NC(=O)NC3CCCCC3)cc1)C(=O)C2(C)C</smiles>

Figure 1: Gliquidone.

\section{EXPERIMENTAL}

\section{Instrumentation and chromatographic conditions}

A Shimadzu HPLC system equipped with LC-10 AT VP pump, SPD-10 AV VP UV-vis detector utilizing a Nucleosil $\mathrm{C}_{18}(10 \mathrm{~mm}, 25 \mathrm{~cm} \times 0.46 \mathrm{~cm})$ column. The chromatographic and integrated data were recorded using a CBM102 Shimadzu. Shimadzu Class-GC 10 software (version 2) was used for data acquisition. Wavelength was monitored at $225 \mathrm{~nm}$ and the injection volume was $10 \mu \mathrm{L}$. The mobile phase, methanol: water (90:10), pH 3.50 was pumped at flow rate of $1 \mathrm{mLmin}^{-1}$ and methylparaben was used as an internal standard.

Chemicals and reagents

Pharmatec (Private) Limited Karachi kindly gifted gliquidone reference standard. Glurenor ${ }^{\mathbb{B}}$ tablets, Pharmatec (Private) Limited labeled to contain 30 mg gliquidone drug and methylparaben were purchased from market. HPLC grade methanol was purchased from Merck Germany. Other chemicals used were of analytical grade.

Preparation of standard and sample solutions

Standard stock solutions of $100 \mathrm{mgmL}^{-1}$ of gliquidone and methylparaben (internal standard) in methanol were prepared in separate volumetric flasks. 2.5 $\mathrm{mL}\left(10 \mu \mathrm{gmL}^{-1}\right)$ of internal standard was added in a series of $25 \mathrm{~mL}$ volumetric flasks and different aliquots of gliquidone standard solution were added to attain concentration range of $0.60,0.80,2.0,5.0,8.0$ and $25 \mu \mathrm{gmL}^{-1}$ using the same solvent as diluent. To determine the accuracy and precision, samples were prepared by spiking reference standards in blank excipients mixture and three replicates of five different concentrations $\left(0.8,2,5,8,10 \mu \mathrm{gmL}^{-1}\right)$ of quality control samples were analyzed on four different days. Accuracy was determined at five concentration levels within the range of calibration i.e near the LOQ, in the middle of the calibration range and so on. Intra-day precision is expressed as a coefficient of variation of samples at the same concentration levels as in accuracy determination. Inter-day precision was determined by analyzing the same set of samples on four different days.

Procedure for Glurenor ${ }^{\circledR}$ tablets

Twenty accurately weighed tablets were crushed to a fine powder. The average mass per tablet was determined. Portion equivalent to $30 \mathrm{mg}$ of gliquidone was transferred accurately into a $100 \mathrm{~mL}$ calibrated flask containing methanol. The content of the flask was shaken for about 60 minutes and diluted to volume with the same solvent, this solution was filtered through Wattmann filter paper to separate out the insoluble excipients and further dilutions were carried out to obtain desire concentration. Final solutions were filtered through a $0.45 \mu \mathrm{m}$ Millipore filter prior to inject in HPLC.

Drug-serum solutions

To $3 \mathrm{~mL}$ of serum, $30 \mathrm{~mL}$ of acetonitrile was added and was vortexed for $1 \mathrm{~min}$ and subsequently centrifuged for $10 \mathrm{~min}$ at $10,000 \mathrm{rpm}$. Supernatant was filtered through $0.45 \mu$ pore size membrane filter. Serum thus obtained was mixed in ratio of $1: 1$ with drug solutions and then serially diluting it with blank serum to attain the desired concentration range 0.6 to $25 \mu \mathrm{gL}^{-1}$ and stored at 
$-20^{\circ} \mathrm{C} .10 \mu \mathrm{L}$ volume of each sample was injected and chromatographed under above conditions.

\section{RESULTS AND DISCUSSION}

According to FDA guidelines commercially available reagents should be used as an internal standard in validation procedures [10]. The internal standard technique is based on the hypothesis that instrumental variations (in injection volume, flow-rate and temperature), sample preparation errors and minor fluctuations of retention times can be compensated [11]. Methyl paraben is not used in the formulation and it was a good choice to be used as internal standard. So in the present study, gliquidone and commercially available and inexpensive methyl paraben as an internal standard were extracted from serum. The two analytes were detected at the same wavelength on UV analysis. Both peaks were well resolved and no interfering peaks were observed during the run time. Representative chromatogram is shown in figure 2.

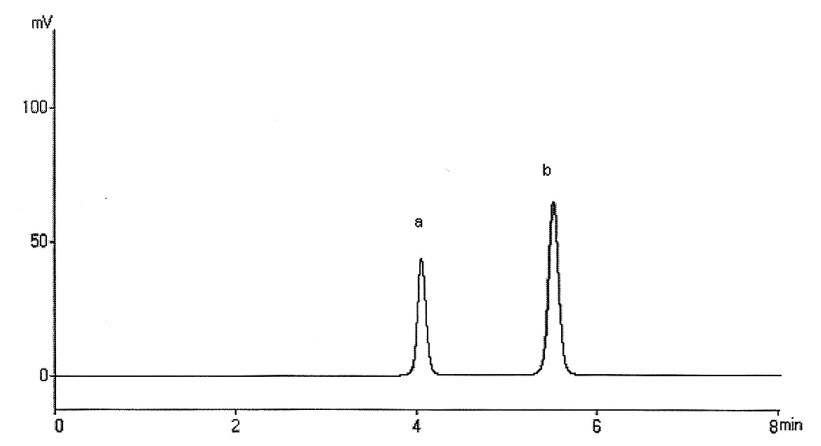

Figure 2: A representative chromatogram of gliquidone (b) and internal standard (a)

\section{Method validation}

The method was validated for the parameters like specificity, range and linearity, limit of detection (LOD), limit of quantitation (LOQ), accuracy and precision. In addition, system suitability parameters were also calculated. The linearity of this method was proved using linear correlation of the peak area values and appropriate concentrations of gliquidone in a range of $0.6-25 \mu \mathrm{gL}^{-1}$. The correlation coefficient of this dependence was calculated to be 0.9999 . The intra and inter day precisions of method were determined to be 1.14 and $1.32 \%$, respectively.

System suitability and specificity

System suitability tests are an integral part of chromatographic methods and are used to verify the resolution and reproducibility of the system. Specificity is the ability to assess unequivocally the analyte in the presence of components that may be expected to be present in the sample matrix [12]. In order to determine the specificity of the method in presence of pharmacopoeial impurities, no peak of excepients was found in chromatogram, which proved that the method can be applied successfully to dosage formulation and method demonstrated good resolutions i.e. 3.19. Specificity was also determined by screening sample of human serum, which was free from interfering endogenous plasma components. Figure 3 represents typical chromatograms of drug and internal standard in serum. Analytical recovery experiments were performed by adding known amount of pure drug to the samples of commercial dosage form. The percent analytical recovery was calculated by comparing concentration obtained from the spiked samples with actual added concentration (table 1).

Table 1

Results of recovery studies of gliquidone

\begin{tabular}{ccc}
\hline $\begin{array}{c}\text { Spiked samples } \\
\left(\mu \mathrm{gmL}^{-1}\right)\end{array}$ & $\begin{array}{l}\text { \%Recovery } \\
(\text { Mean })\end{array}$ & \%RSD \\
\hline 0.6 & 100.87 & 1.90 \\
0.8 & 100.10 & 1.30 \\
2.0 & 100.04 & 1.36 \\
5.0 & 98.94 & 1.80 \\
8.0 & 99.58 & 1.37 \\
25 & 100.00 & 1.77 \\
\hline
\end{tabular}

${ }^{*}$ Relative standard deviation

Linearity, limit of detection and limit of quantification

The linearity of an analytical method is its ability to elicit test results that are directly, or by a well defined mathematical transformation, proportional to the concentration of analyte in samples within a given range [12]. The linearity of the method was observed in the expected concentration range demonstrating its suitability for analysis. To evaluate the linearity of the method, different dilutions were made from the standard stock solutions in the working range of $0.60,0.80,2.0,5.0,8.0$ and $25 \mu \mathrm{gmL}^{-1}$. A curve relating the ratio of the peak areas of gliquidone to the concentration of gliquidone evaluated was $y=23497 x+1084$ with correlation coefficient of 0.9999 . LOD and LOQ were calculated using equation 1 and 2 , respectively.

$3.3 \sigma$

$$
\mathrm{LOD}=
$$

Where $\sigma$ is the standard deviation of the response, $\mathrm{S}$ is the slope of the calibration curve. The LOQ for this assay was $0.60 \mu \mathrm{gmL}^{-1}$ and LOD was $0.03 \mu \mathrm{gmL}^{-1}$.

Repeatability, intra and inter-day precision and accuracy

The precision of the method was investigated with respect to repeatability. Repeatability of the method was checked by analyzing six replicate samples of gliquidone and calculating relative standard deviation (\%RSD). To determine intermediate precision, standard solutions of gliquidone were analyzed three times within the same day (intra day variation) and on four different days (inter day variation) using the same chromatographic system and the same column by two analysts on a different day. The assay result indicated that the method was capable with high precision. The accuracy of an analytical method is the closeness of test results obtained by that method to the true value [12]. To determine accuracy of the method, working standard of gliquidone was prepared in triplicate at different concentration levels and analyzed. The results of accuracy studies are shown in table 2 and it is evident that the method was accurate. The accuracy of the presented method in serum was also confirmed by the recovery studies. The recovery data expressed in tables 3 shows that the method is accurate for determination of gliquidone in human serum.

Table 2

Intraday and inter day precision and accuracy

\begin{tabular}{|c|c|c|c|c|c|c|c|c|}
\hline \multirow{2}{*}{$\begin{array}{l}\text { Spiked } \\
\left(\mu g m L^{-1}\right)\end{array}$} & \multicolumn{2}{|l|}{ Day 1} & \multicolumn{2}{|c|}{ Day 2} & \multicolumn{2}{|c|}{ Day 3} & \multicolumn{2}{|c|}{ Day 4} \\
\hline & $\%$ Recov & $\%$ RSD & $\%$ Recov & $\%$ RSD & $\%$ Recov & $\%$ RSD & \%Recov & $\%$ RSD \\
\hline 0.80 & 99.92 & 1.38 & 102.78 & 0.94 & 100.08 & 1.12 & 99.23 & 1.76 \\
\hline 2.00 & 99.84 & 1.45 & 100.64 & 1.14 & 100.07 & 1.78 & 99.88 & 1.64 \\
\hline 5.00 & 99.93 & 1.19 & 100.14 & 1.75 & 100.00 & 1.69 & 99.24 & 1.45 \\
\hline 8.00 & 100.00 & 0.56 & 100.00 & 1.33 & 100.00 & 1.15 & 100.00 & 1.49 \\
\hline 10.0 & 99.36 & 1.16 & 99.77 & 0.65 & 100.45 & 1.73 & 100.10 & 1.69 \\
\hline
\end{tabular}


Application in human serum

Serum samples were spiked with gliquidone and internal standard at different concentration levels and assayed. Obtained recoveries and coefficient of variation are showed that no considerable differences exist between mean recoveries of gliquidone in serum sample. Thus, serum did not interfere in the estimation of gliquidone (figure 3). The recovery values of gliquidone (table 3 ) in human serum clearly indicates the applicability of the present method for the required purpose.

Table 3

Results of the proposed method for estimation of gliquidone in human serum

\begin{tabular}{lll}
\hline $\begin{array}{l}\text { Amount of drug } \\
\text { added }(\mathrm{mg})\end{array}$ & \%Recovery & \%RSD \\
\hline 0.60 & 99.97 & 1.71 \\
0.80 & 99.89 & 1.61 \\
2.00 & 100.12 & 1.77 \\
5.00 & 99.10 & 1.23 \\
8.00 & 98.09 & 1.57 \\
25.0 & 101.0 & 1.82 \\
Correlation coefficient & 0.9981 & \\
\hline
\end{tabular}

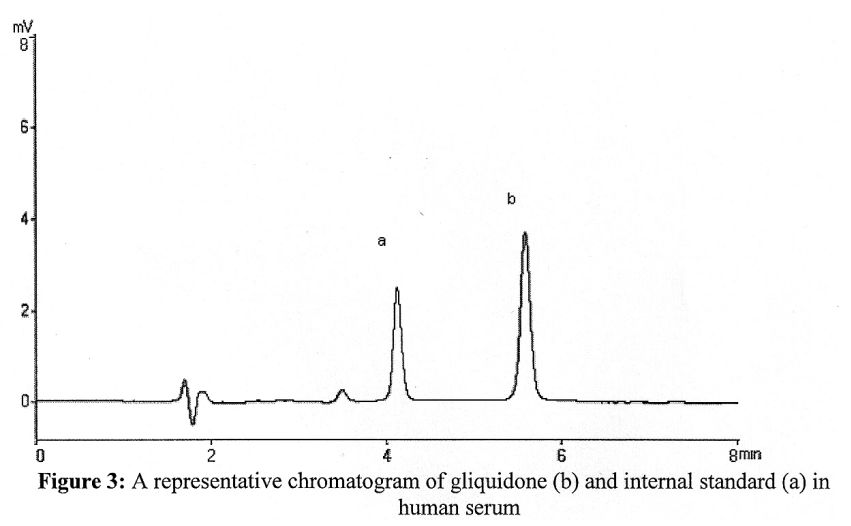

\section{CONCLUSION}

A fully validated, simple and reliable RP-HPLC procedure for the assay of gliquidone in formulation and in human serum has been developed for the first time. Hence, it can be recommended for the routine quality control of this antidiabetic drug. The availability of the internal standard (methylparaben), the simplicity of the separation procedure and the low limit of quantification makes this method suitable for quick and routine analysis. The assay has a lower detection limit of $0.03 \mu \mathrm{gmL}^{-1}$ of gliquidone. The developed method is accurate, precise and linear within the desire range.

\section{REFERENCE}

1. The Merck Index Merck and Co Inc. White House Station, NJ, USA: Published on CD-ROM by Chapman and Hall / CRC. Hampden data services Ltd.; Monograph No. 4452, 1999.

2. K. Parfitt. Martindale, The Complete Drug Reference, London, Pharmaceutical Press, $32^{\text {nd }}$ edition, 1999.

3. British Pharmacopoeia, The Stationary office, London, Volume III 2005

4. II. Dedov, IIu. Demidova, S. V. Pisklakov, E. A. Antokhin, Probl Endokrinol (Mosk). 39, 6-8, (1993).

5. M. S. Arayne, N. Sultana, A. Z. Mirza, Pak. J. Pharm. Sci. 19, 185, (2006).

6. H. H. Maurer, C. Kratzsch, T. Kraemer, F. T. Peters, A. A. Weber, $J$ Chromatogr B Analyt Technol Biomed Life Sci. 773, 63 (2002).

7. P. Guo, Z. W. Li, C. H. Chen, S. P. Deng, S. G. Tang, Yao Xue Xue Bao. 27, 452, (1992).

8. H. von Nicolai, R. Brickl, H. Eschey, A. Greischel, G. Heinzel, E. Konig, J. Limmer, E. Rupprecht, Arzneimittelforschung, 47, 247, (1997).

9. S. Sridevi, P. V. Diwan, Pharmacy and Pharmacology Communications. 6, $303,(2000)$.

10. Guideline for submitting samples and analytical data for methods validation, Food and Drug Administration, Center for Drugs and Biologics, Office of Drug Research and Review (HFN-100), 5600 Fishers Lane. Rockville, Maryland (1987).

11. J. Wieling, P. M. J. Coenegracht, C. K. Mensink, J. H. G. Jonkman and D. A. Doornbos, Journal of Chromatography A, 594, 45, (1992).

12. United States Pharmacopeia/National Formulary, USP 30, NF 25 , Rockville, 2007. 